\title{
Predictores de una interacción eficaz entre madre e hijo/a en contextos de riesgo psicosocial $^{*}$
}

\section{Predictors of an Efficient Interaction between Mother and Child in Psychosocial Risk}

Recepción: 31 Julio 2013 | Aprobación: 23 Enero 2017

\author{
Miriam Álvarez Lorenzo \\ Universidad de La Laguna, España \\ María José Rodrigo López \\ Universidad de La Laguna, España \\ Guacimara Rodríguez SuÁrez \\ Universidad de La Laguna, España
}

a Autor de correspondencia. Correo electrónico: malore@ull.es

\section{RESUMEN}

Este estudio analiza la influencia de las prácticas, las metas, la representación materna de las propias acciones y la predictibilidad de prácticas y metas según los hijos/as sobre la interaccion madre-hijo/a observada en una tarea colaborativa. Las participantes fueron 82 diadas en riesgo psicosocial. Las madres reportaron las prácticas y las metas en situaciones hipotéticas y la representación de la propia acción en la interacción; los hijos/as, la predictibilidad de las prácticas y las metas de las propias madres. Los modelos de regresión muestran la influencia negativa de las prácticas coercitivas y restrictivas, la influencia positiva de la representación de la acción y de la predictibilidad de prácticas y metas en la dinámica de la interacción madre-hijo/a.

Palabras clave

predictibilidad de prácticas y metas; representación de la acción; riesgo psicosocial; interacción madre-hijo.

Para citar este artículo: Álvarez Lorenzo, M., Rodrigo López, M. J., \& Rodríguez Suárez, G. (2017). Predictores de una interacción eficaz entre madre e hijo/a en contextos de riesgo psicosocial. Universitas Psychologica, 16(1) 1-10. http://dx.doi.org/10.11144/J averiana.upsy16-1.piem

\begin{abstract}
This study investigates the influence of the practices, goals, maternal representation of their action and the child's readability of practices and goals on the mother-child interaction observed during a collaborative task. The participants were 82 dyads at psychosocial risk. The mothers reported their practices and goals in hypothetical situations and the representation of their actions in the interaction. Children reported the readability of mothers' practices and goals. The regression models showed the negative influence of the coercitive and restrictive practices, the positive influence of the maternal representation of their child-rearing actions and the child's readability of the practices and goals on the dynamic of the mother-child interaction.

Keywords

readability of practices and goals; representation of child-rearing practices; psychosocial risk; mother-child interaction.
\end{abstract}




\section{Introducción}

La interacción educativa que se desarrolla entre padres e hijos es un engranaje sutil que condiciona el buen ajuste y desarrollo de los hijos. Como señalan los enfoques neovygotskianos, en dichas interacciones se lleva a cabo un proceso de aprendizaje guiado, donde los padres u otros agentes de socialización apoyan y modelan la adquisición de destrezas y conocimiento en los hijos, durante la realización conjunta de tareas (Gauvain \& Pérez, 2007; Rogoff,1990). Durante este proceso, los niños aprenden lo que es importante para los adultos e igualmente a desarrollar actividades significativas para su grupo, a la vez que adquieren un conocimiento del mundo relevante para la familia, la escuela o la sociedad. Es también importante que durante esas interacciones se creen relaciones de confianza y de cooperación mutua con los padres, ayudando así a un clima favorable para el sostenimiento de una interacción eficaz y sostenida en el tiempo (Grusec \& Davidov, 2010).

Sin embargo, es posible que la adecuada regulación de esa participación conjunta en tareas no se lleve a cabo del mismo modo en todos los contextos familiares. En aquellas familias en situación de riesgo psicosocial, donde son frecuentes los momentos de estrés y la preocupación de la familia se centra en otros aspectos, podrían no darse las condiciones que favorecen una interacción eficaz y una estimulación adecuada del desarrollo del hijo/a. Por ello, el propósito de este estudio es analizar la eficacia de las interacciones que se llevan a cabo en la díada madre-hijo/a de este tipo de familias, entre la segunda infancia y preadolescencia, así como los posibles predictores de esta interacción.

Entre los predictores posibles, las prácticas y metas educativas podrían influir en la calidad de la interacción madre-hijo/a. En familias normalizadas, se ha observado que las madres que muestran más autoridad generan menos interacciones cooperativas y sensibles con sus hijos/as que las madres que muestran mayor reciprocidad en sus relaciones educativas (Bugental \& Grusec, 2006). Las prácticas basadas en la sensibilidad y responsividad se relacionan con interacciones en las que los padres muestran a sus hijos su afecto, su aceptación, les dan apoyo y se comunican para razonar con ellos (Martínez \& García, 2007). En cuanto a las familias en riesgo psicosocial, los padres suelen desarrollar prácticas educativas relacionadas con la permisividad, la negligencia y la coerción (Rodrigo, Byrne, \& Rodríguez, 2013; Rodrigo, Máiquez, Martín, \& Byrne, 2008). A la hora de establecer interacciones con sus hijos/as, las madres con abuso físico suelen mostrar conductas basadas en el poder físico y la amenaza verbal, con muestras de ira, rechazo e impulsividad (Azar, 2002; Bousha \& Twentyman, 1984; Cicchetti \& Toth, 2015; Egeland, Pianta, \& O'Brien, 1993; Howes, Cicchetti, \& Toth, 2000), bajos niveles de afectividad y prácticas incoherentes y severas (Conger et al., 2002). Por otro lado, las madres negligentes se caracterizan por dar menos instrucciones a sus hijos/as, tener un menor nivel de actividad y estimular menos a sus hijos durante la interacción (Bousha \& Twentyman,1984; Crittenden, 1981). Por tanto, estas prácticas, como elemento fundamental de los contextos de interacción, pueden incidir en la calidad del mismo, y como consecuencia en el desarrollo de los niños (Bernier, Calkins, \& Bell, 2016; Orozco-Hormaza, Sánchez-Ríos, \& Cerchiaro-Ceballos, 2012).

En cuanto a las metas educativas, diversos estudios han encontrado que las madres que maltratan a sus hijos plantean metas parentales a corto plazo y centradas en sus propios intereses (Grusec \& Walters, 1991), aunque no se ha investigado cómo influyen estas en las interacciones con sus hijos/as.

Hay otros predictores menos estudiados que también podrían estar relacionados con la calidad de las interacciones madre-hijo/a. Los nuevos modelos bidireccionales de socialización consideran la relación padres-hijos como un producto de las acciones llevadas a cabo en un continuo de interacciones donde unos y otros se influyen mutuamente (Barbot, Crossman, Hunter, Grigorenko, \& Luthar, 2014; Kuczynski, 2003; Kuczynski \& De Mol, 2015; Maccoby, 2003). Por ende, es importante tener en cuenta 
tanto la representación de la madre de su acción educativa durante la interacción como la predictibilidad de las prácticas y metas maternas por parte de los hijos/as. Ambas variables pueden ser explicativas de los procesos que subyacen al desarrollo de la interacción. Vamos a analizarlas en más detalle.

La representación de la acción se refiere a cómo caracterizan las madres y los padres las acciones educativas que están llevando a cabo con sus hijos/as, la cual puede tener diversos niveles de complejidad, ya que puede ir desde la descripción simple y literal de lo que hacen hasta una más compleja que implica comprensión más amplia y abstracta centrada en las consecuencias a corto y largo plazo de sus acciones (Rodrigo, Janssens, \& Ceballos, 2001; Vallacher \& Wegner, 1985). Investigaciones con familias normalizadas han señalado que las madres que manifiestan niveles altos de representación de sus acciones educativas son más cálidas hacia sus hijos y menos restrictivas, mostrando menos presión y menos directividad durante una tarea de colaboración (Rodrigo et al., 2001). En cambio, en las familias en riesgo, las madres suelen utilizar exposiciones literales de sus acciones o meras descripciones de sus acciones, con las consecuencias a corto plazo, lo que influye en la peor calidad de las interacciones en tareas conjuntas con el hijo/a (Rodríguez, Rodrigo, Janssens, \& Triana, 2011).

En cuanto a la predictibilidad tiene que ver con la percepción que hijos e hijas tienen de las metas y las prácticas de sus padres. El modelo propuesto por Grusec y Goodnow (1994) postula que el proceso por el cual los hijos/ as interiorizan los mensajes educativos depende de en qué medida este es fácilmente predecible por parte del niño. Rodrigo, Janssens y Ceballos (1999) mostraron que cuando los hijos eran capaces de predecir adecuadamente los mensajes educativos de los padres, también lo eran de mostrar un mayor grado de autorregulación durante una tarea conjunta. Este proceso parece no desarrollarse de igual forma en las familias en situación de riesgo psicosocial. Álvarez, Rodrigo y Rodríguez (2013), en un estudio realizado con madres e hijos/as en riesgo psicosocial, encontraron que las prácticas de coerción y negligencia contribuyen a disminuir el grado de predictibilidad, mientras que las negligentes tampoco facilitan la predictibilidad de la meta educativa de la madre.

En suma, aunque tradicionalmente se ha estudiado la influencia de las metas y las prácticas sobre la calidad de las interacciones, en esta investigación se va a analizar si también la representación materna de sus acciones y la predictibilidad de las metas y prácticas según los hijos/as contribuyen a explicarla. Entre las dimensiones de la interacción se ha optado por analizar tanto la participación activa de ambos miembros de la díada que llevaría a un sostenimiento de la interacción como la pasividad de la díada que conduciría a bloqueos e interrupciones en el proceso interactivo. La razón de ello es que en las familias en riesgo es muy frecuente el desinterés o la falta de atención al niño en situaciones de aprendizaje, lo cual podría explicar su mayor propensión a tener retrasos en su desarrollo. De hecho, se ha elegido a niños de segunda infancia y preadolescencia, teniendo en cuenta que es un período menos estudiado y porque a dichas edades aumentan todavía más dichos retrasos evolutivos. En particular, se espera que un uso más frecuente de las metas y practicas más positivas (menos negligentes y coercitivas) así como un mayor nivel de representación de la acción educativa por parte de las madres y una mayor predictibilidad de las prácticas y metas por parte de los hijos, van a contribuir a una mayor participación y menor pasividad tanto en la madre como en el hijo/a durante la realización conjunta de una tarea.

\section{Método}

\section{Participantes}

Participaron 82 diadas de madres (edad media $=36.26 ; \mathrm{DE}=6.86$ ) con un hijo o hija (62 \% niños y $37 \%$ niñas) de 8 a 12 años $($ media $=10, \mathrm{DE}=1.38)$. El $56.7 \%$ viven en zona urbana, la mayoría (70 \%) tienen estudios primarios incompletos, están desempleadas (60.5 
\%) y son familias monoparentales (50.7\%). Para ello, se solicitó a los Servicios Sociales de diferentes municipios de la isla de Tenerife que remitieran familias con menores en situación de riesgo de desprotección por la presencia de pautas coercitivas y/o negligentes por parte de los padres, y que además los menores estuvieran en el rango de edad requerido para una mayor capacidad cognitiva de respuesta a las situaciones hipotéticas y de resolución de la tarea interactiva (Rodrigo et al., 1999).

\section{Instrumentos}

\section{Instrumento de metas y prácticas educativas}

Se diseñaron nueve situaciones hipotéticas conflictivas, tres de comportamientos externalizantes del hijo/a (v. g. "Su hijo/tú se está peleando y pegando con otro/a niño/a por un juguete que los/as dos quieren"), tres internalizantes del hijo/a (v. g., "Su hijo/tú llega un día a casa del colegio, parece muy triste y no quiere hablar con nadie") y tres que violan las normas convencionales (v. g., "Su hijo/tú siempre llega a casa más tarde de la hora que usted le ha dicho"). A los hijos se les presentaban las mismas situaciones que a sus madres. Ante estas situaciones, las madres debían reportar lo que hacían y los hijos, por su parte, lo que hacían las madres, con el fin de analizar posteriormente el grado de coincidencia entre unas respuestas y otras. Para analizar las prácticas educativas se les preguntaba a las madres y a los hijos de manera abierta: "¿Qué harías/qué haría tu madre en esa situación?”. Para la madre, se presentaron además una serie de alternativas sobre diferentes prácticas, para que evaluara la frecuencia de uso de cada una en cada situación. A partir del análisis de las repuestas abiertas de la madre y del hijo/a se construyeron las siguientes categorías: Coercitiva (v. g., madre: "le digo que se merece un cachete"; hijo: "me pega porque soy tonto"), Inductiva: (v. g., madre: "intento que entienda lo importancia de comunicarse"; hijo: "me enseña a hablar con mis amigos"), Restrictiva: (v. g., madre: "le castigo"; hijo: "no me deja jugar a la play-station"), Permisiva (v. g., madre: "me resigno"; hijo: "me deja hacer lo que quiero"), Negligente (v. g., madre: "no le hago caso"; hijo: "pasa de mî"). Los porcentajes de acuerdo entre jueces oscilaron entre el $95 \%$ y el $99 \%$ y los índices Kappa promedio fueron de 0.82 para la madre y 0.81 para el hijo/a.

Para analizar las metas educativas, se preguntaba a la madre y al hijo/a: "Con su forma de actuar iqué quiere lograr de su hijo/a? iqué quiere conseguir tu madre de ti?". A partir de las respuestas, se creó el siguiente sistema de categorías: Control (v. g., madre: "que pare de hacer lo que está haciendo"; hijo: "que no lo haga más”), Desarrollo Personal (v. g., madre: "que tenga confianza en sí misma"; hijo: "quiere que sea feliz"), Normas (v. g., madre: "que se porte bien", hijo: "que llegue puntual"), Afecto (v. g., madre: "que confíe en nosotros"; hijo; "que sienta que me quiere"). Los porcentajes de acuerdo oscilaron entre el $85 \%$ y el $90 \%$ y los índices de Kappa fueron de 0.79 para la madre y 0.81 para el hijo/a.

Para calcular la medida de la predictibilidad de las metas y prácticas educativas, esto es, la precisión con que la respuesta del niño se aproxima a la de la madre, se llevó a cabo el siguiente procedimiento. Para las prácticas, se compararon las respuestas de los niños con las respuestas abiertas y cerradas de la madre, asignando la puntuación de 0 si el niño/a no sabe predecir cuál es la práctica de la madre y 4 puntos si existe una concordancia total, mientras que las puntuaciones intermedias se asignan en función del grado de semejanza de las respuestas madre e hijo. Respecto a la predictibilidad de las metas educativas, se siguió el mismo sistema. Los dos jueces evaluaron la precisión de las respuestas, obteniendo unos índices Kappa de 0.82 para la predictibilidad de prácticas y un 0.81 para la predictibilidad de metas.

\section{La tarea de los recados}

Para el análisis de las conductas interactivas materno-filiales, se grabó en video a la díada interactuando durante la "tarea de los recados" 
elaborada por Radziszewska y Rogoff (1988). La díada debía trazar la ruta más corta para hacer una serie de recados, siguiendo un mapa de una ciudad imaginaria para preparar una excursión (v. g., comprar bocadillos, sacar dinero del banco, etc.). Siguiendo el sistema de categorías de Rodrigo et al. (2001), se seleccionó para este estudio, tanto para la madre como para el hijo/ a, las categorías de pasividad (desentenderse de la tarea, no hacer nada, no mostrar iniciativas) y comentarios sobre la tarea (hablar con el otro sobre la estrategia utilizada, sobre los planes a seguir), dado que se quería investigar el nivel de regulación de la actividad. Dos observadores codificaron dichas categorías en una escala de 0 a 6 puntos (desde no fue observado a fue observado con mucha frecuencia). Los resultados de la prueba Kappa de fiabilidad interobservadores fueron para la madre y el hijo, respectivamente: pasividad: $0.72,0.73$ y comentarios sobre la tarea: $0.75,0.79$.

Entrevista post-video para el análisis de la representación de la propia acción educativa

Al finalizar cada grabación, se le presentaban a la madre seis escenas del vídeo en las aparecían haciendo o diciendo algo a sus hijos/as. En cada uno de esos momentos se le preguntaba a la madre "qué" estaba haciendo y "por qué" estaba haciendo eso, con el fin de obtener el nivel de representación de sus acciones educativas (Rodrigo et al., 2001). Las respuestas se clasificaron en cinco niveles de complejidad: Nivel 1) descripción de la acción física: las madres enuncian sus propias acciones de forma concreta y simple sin mencionar las metas (v. g., "Estaba haciendo una raya con el lápiz); Nivel 2) Descripción de la acción con metas inmediatas: las madres formulan la acción de forma concreta y simple, pero contiene una meta o plan inmediatos (v. g., "estaba buscando el mejor camino para llegar a la panadería"); Nivel 3) Descripción de la interacción física: las madres refieren la interacción con su hijo de una forma concreta y simple, sin mencionar ninguna meta (v. g., "Le estaba dando el lápiz a mi hijo");
Nivel 4) Descripción de la acción con metas inmediatas: las madres exponen la interacción de forma concreta y simple, pero incluyen una meta inmediata (v. g., "Estábamos decidiendo cuál era el recorrido más corto para hacer los recados que nos quedan"); Nivel 5) Metas de interacción a largo plazo: las madres realizan una explicación de la interacción compleja, que incluye un plan estratégico y metas a medio y largo plazo (v. g., "Estaba dejando que mi hijo pensara el recorrido para que aprenda a organizarse por sí mismo").

El nivel de representación se basa pues en la media de puntuaciones (1 a 5) a las doce preguntas de "qué" y "por qué" recogidas en los seis momentos en los que se detuvo el vídeo. Cada respuesta fue codificada de forma independiente por dos jueces ("iQué hace en este momento?": Kappa = 0.89; "iPor qué lo hace?": Kappa $=0.91)$.

\section{Procedimiento}

Los profesionales de los servicios sociales contactaron con las familias que, tras conocer el estudio, firmaron su consentimiento según el protocolo del Comité de Ética de la Universidad de La Laguna. A continuación, se citaron a las familias en los locales de los servicios para poder realizar las entrevistas por separado para las madres y para los hijos e hijas. Luego, se llevó a cabo la grabación de la interacción cuando la madre y el hijo/a desarrollaban la tarea de los recados. Al finalizar la grabación, el video fue presentado a las madres y se midió el nivel de representación de la acción.

\section{Resultados}

Se diseñaron modelos de regresión con las variables de metas, prácticas, nivel de representacion de la acción y predictibilidad de las prácticas y metas sobre las acciones observadas en los vídeos, tanto de la madre como del niño/a. Con el fin de que los modelos fueran parsimoniosos y que cumplieran los requisitos de linealidad, normalidad y homocedasticidad, se calcularon correlaciones bivariadas entre las 
variables del estudio, mediante el coeficiente de correlación de Pearson, tanto para la madre como para el hijo/a. A partir de estos análisis, se pusieron a prueba cuatro modelos de regresión lineal jerárquica entre las variables predictoras de metas y prácticas de las madres, el nivel de representación de la acción y la predictibilidad de prácticas y metas sobre los comentarios y la pasividad durante la interacción en la tarea de los recados, tanto para la madre como para el hijo/ a. Todas las variables incluidas en los modelos de regresión fueron estandarizadas con el fin de evitar que las distintas escalas de las variables pudieran influir en los resultados del análisis, siguiendo las recomendaciones de Tabachnick y Fidell (2007). En consonancia con el objetivo de este estudio, se utilizó el procedimiento por pasos: en el primero, se introdujeron las prácticas o metas de la madre y el nivel de representación de la acción y en el segundo, la predictibilidad de metas y práctica porque se esparaba que tuvieran poder predictivo una vez incluido el efecto de las variables tradicionales. Para la interpretación de la significación global del modelo, se examinó el estadístico $F$, los valores de la $R^{2}$ y la $R^{2}$ de cambio y la contribución específica de cada dimensión a la varianza total, explicada por el modelo a través de la significatividad y el valor de la correlación semiparcial al cuadrado, $r s^{2}$.

En primer lugar, el modelo de regresión sobre la conducta de pasividad de la madre, resultó significativo, $F(6.74)=4.64 ; p<0.001$, explicando en total un $32 \%$ de la varianza. El valor de las $R^{2}$ de cambio pone de manifiesto que las medidas de predictibilidad de prácticas y metas añadieron a las prácticas y al nivel de representación de la acción el $9 \%$ de la varianza explicada. Las prácticas inductivas y la predictibilidad de las prácticas son las variables que contribuyeron negativamente a las conductas de pasividad de la madre, mientras que las prácticas coercitivas lo hicieron de forma positiva (Tabla 1 ).

\section{TABLA 1}

Modelo de regresión sobre la pasividad de la madre en la tarea de los recados

\begin{tabular}{|c|c|c|c|c|}
\hline & \multicolumn{4}{|c|}{ Pasividad de la madre } \\
\hline & $\beta$ & $r s^{2}$ & $\operatorname{AdjR}^{2}$ & $\Delta R^{2}$ \\
\hline Paso 1 & & & 0.25 & \\
\hline Prácticas Inductivas & -0.26 & 0.038 & & \\
\hline Prácticas Coercitivas & $0.38 *$ & 0.099 & & \\
\hline Prácticas Restrictivas & 0.04 & 0.001 & & \\
\hline Nivel de representación de la acción & -0.04 & 0.001 & & \\
\hline Paso 2 & & & 0.32 & 0.09 \\
\hline Prácticas Inductivas & $-0.35^{*}$ & 0.061 & & \\
\hline Prácticas Coercitivas & $0.34 *$ & 0.08 & & \\
\hline Prácticas Restrictivas & 0.06 & 0.003 & & \\
\hline Nivel de representación de la acción & -0.01 & 0 & & \\
\hline Predictibilidad de prácticas & $-0.29 *$ & 0.067 & & \\
\hline Predictibilidad de metas & -0.04 & 0.001 & & \\
\hline
\end{tabular}

El modelo de regresión sobre la conducta de comentarios de la madre, resultó significativo, $F(6.74)=4896 ; p<0.001$, explicando en total un $29 \%$ de la varianza. El valor de las $R^{2}$ de cambio indica que las medidas de predictibilidad de prácticas y metas añadieron a las prácticas y al nivel de representación de la acción el $10 \%$ de la varianza explicada. Las prácticas coercitivas y restrictivas son las que contribuyeron negativamente a la predicción de las conductas de comentarios de las madres en la interacción con su hijo, mientras que la predictibilidad de las prácticas predijo positivamente la realización de los comentarios de la madre (Tabla 2).

\section{TABLA 2}

Modelo de regresión sobre los comentarios de la madre en la tarea de los recados

\begin{tabular}{|c|c|c|c|c|}
\hline & \multicolumn{4}{|c|}{ Comentarios de la madre } \\
\hline & $\beta$ & $r s^{2}$ & $\operatorname{Adj} R^{2}$ & $\Delta R^{2}$ \\
\hline Paso 1 & & & 0.22 & \\
\hline Prácticas Inductivas & 0.19 & 0.03 & & \\
\hline Prácticas Coercitivas & $-0.36 * *$ & 0.124 & & \\
\hline Prácticas Restrictivas & $-0.29 *$ & 0.075 & & \\
\hline Nivel de representación de la acción & 0.25 & 0.06 & & \\
\hline Paso 2 & & & 0.29 & 0.1 \\
\hline Prácticas Inductivas & 0.2 & 0.048 & & \\
\hline Prácticas Coercitivas & $-0.36^{* *}$ & 0.124 & & \\
\hline Prácticas Restrictivas & $-0.34 * *$ & 0.108 & & \\
\hline Nivel de representación de la acción & 0.24 & 0.052 & & \\
\hline Predictibilidad de prácticas & $0.3^{*}$ & 0.073 & & \\
\hline Predictibilidad de metas & 0.06 & 0.003 & & \\
\hline
\end{tabular}


Como se puede apreciar en la Tabla 3, se incluyen los resultados del modelo de regresión de las prácticas, la representación de la acción y la predictibilidad de metas y prácticas sobre la conducta de pasividad del hijo/a. Este modelo resultó significativo, $F(5.75)=5.175 ; p<$ 0.001, explicando en total un $32 \%$ de la varianza. El valor de las $R^{2}$ de cambio pone de manifiesto que las medidas de predictibilidad de prácticas y metas añadieron a las prácticas y al nivel de representación de la acción el $8 \%$ de la varianza explicada. La práctica coercitiva contribuyó positivamente a las conductas de pasividad del hijo, mientras que el nivel de representación de la acción y la predictibilidad de las metas, lo hicieron de forma negativa.

Como se puede apreciar en la Tabla 3, se incluyen los resultados del modelo de regresión de las prácticas, la representación de la acción y la predictibilidad de metas y prácticas sobre la conducta de pasividad del hijo/a. Este modelo resultó significativo, $F(5.75)=5.175 ; p<$ 0.001, explicando en total un $32 \%$ de la varianza. El valor de las $R^{2}$ de cambio pone de manifiesto que las medidas de predictibilidad de prácticas y metas añadieron a las prácticas y al nivel de representación de la acción el $8 \%$ de la varianza explicada. La práctica coercitiva contribuyó positivamente a las conductas de pasividad del hijo, mientras que el nivel de representación de la acción y la predictibilidad de las metas, lo hicieron de forma negativa.

TABLA 3

Modelo de regresión sobre la pasividad del hijo/a en la tarea de los recados

\begin{tabular}{lllll}
\hline & \multicolumn{4}{l}{ Pasividad del hijo/a } \\
\cline { 2 - 5 } & $\beta$ & $r s^{2}$ & $A d j R^{2}$ & $\Delta R^{2}$ \\
\hline Paso 1 & $0.27^{*}$ & 0.058 & & \\
Prácticas Coercitivas & 0.06 & 0.003 & & \\
Prácticas Inductivas & $-0.48^{* * *}$ & 0.21 & & \\
Nivel de representación de la acción & & & 0.32 & 0.08 \\
\hline Paso 2 & $0.27^{*}$ & 0.058 & & \\
Prácticas Coercitivas & 0.11 & 0.008 & & \\
Prácticas Inductivas & $-0.42 * *$ & 0.16 & & \\
Nivel de representación de la acción & 0.09 & 0.006 & & \\
Predictibilidad de prácticas & $-0.32 *$ & 0.081 & & \\
Predictibilidad de metas & &
\end{tabular}

$* p<0.05 ; * * p<0.01 ; * * * p<0.001$.

Fuente: elaboración propia
Asimismo, se puso a prueba el modelo de predicción de las metas de la madre sobre la pasividad del hijo, el cual resultó significativo, $F(7.73)=4208 ; p<0.001$, explicando en total un $32 \%$ de la varianza. El valor de las $R^{2}$ en cambio pone de manifiesto que las medidas de predictibilidad de prácticas y metas añadieron a las metas y al nivel de representación de la acción el $10 \%$ de la varianza explicada. Las metas de la madre basadas en las normas, afecto y promoción del desarrollo son las variables que contribuyen positivamente a la pasividad del hijo, mientras que el nivel de representación de la acción y la predictibilidad de las metas lo hicieron de forma negativa (Tabla 4).

\section{TABLA 4}

Modelo de regresión sobre la pasividad del hijo/a en la tarea de los recados

\begin{tabular}{|c|c|c|c|c|}
\hline & \multicolumn{4}{|c|}{ Pasividad del hijo/a } \\
\hline & $\beta$ & $r s^{2}$ & $\operatorname{Adj}^{2}$ & $\Delta R^{2}$ \\
\hline Paso 1 & & & 0.23 & \\
\hline Meta Normas & $0.48^{*}$ & 0.063 & & \\
\hline Meta Control & 0.04 & 0.002 & & \\
\hline Meta Afecto & 0.2 & 0.025 & & \\
\hline Meta Promover el desarrollo & $0.5^{*}$ & 0.075 & & \\
\hline Nivel de representación de la acción & $-0.54 * * *$ & 0.271 & & \\
\hline Paso 2 & & & 0.32 & 0.11 \\
\hline Meta Normas & $0.59 *$ & 0.085 & & \\
\hline Meta Control & 0.12 & 0.01 & & \\
\hline Meta Afecto & $0.31 *$ & 0.053 & & \\
\hline Meta Promover el desarrollo & $0.57^{*}$ & 0.095 & & \\
\hline Nivel de representación de la acción & $-0.46 * * *$ & 0.187 & & \\
\hline Predictibilidad de prácticas & 0.08 & 0.005 & & \\
\hline Predictibilidad de metas & $-0.36^{* *}$ & 0.1 & & \\
\hline
\end{tabular}

\section{Discusión}

En este estudio, se ha podido comprobar que las variables tradicionales de las prácticas y metas tienen un poder predictivo sobre la interacción entre madre e hijo/a. En particular, la coerción de la madre predice que, tanto ella como el hijo/a se muestren pasivos en la interacción. Esta práctica no favorece el andamiaje necesario para el desarrollo del aprendizaje de los/as menores. Este resultado iría en consonancia con los encontrados en otros estudios sobre madres que maltratan, caracterizando a las mismas con el uso de las prácticas severas, de castigo y 
de afirmación de poder (Oldershaw, Walters, \& Hall, 1986; Trickett \& Kuczynski, 1986). Sin embargo, el uso de las prácticas inductivas predice negativamente la pasividad de la madre. Asimismo, tanto las prácticas de coerción como las restrictivas predicen de forma negativa los comentarios de la madre, que son un signo de su implicación en la tarea. Tal como se observa en el video, las interacciones de las madres coercitivas y aquellas que son restrictivas conllevan menos comentarios, lo cual puede dificultar la internalización del mensaje por parte de los hijos/as (Hoffman, 1970; Maccoby, 1992). La aportación más novedosa de este estudio es que también las variables más bidireccionales como la representación de la acción materna y la predictibilidad del niño contribuyen en gran medida a explicar la calidad de las interacciones. En particular, la predictibilidad de las prácticas se asocia negativamente a la pasividad de la madre y del hijo/a y positivamente al uso de comentarios por parte de la madre. Ello significa que cuando los hijos predicen adecuadamente las prácticas de la madre, alcanzándose un alto nivel de acuerdo entre ambos, la madre se muestra más activa en la tarea, realizando más comentarios positivos, los cuales suponen la esencia de un proceso eficaz de enseñanza-aprendizaje. Esta relación ha sido estudiada por otros autores que han encontrado en sus resultados la relación entre el nivel de acuerdo de las percepciones y la calidad de la interacción (Álvarez et al., 2013; Grusec \& Goodnow, 1994; Rodrigo et al., 1999). Por su parte, la predictibilidad de metas influye de forma negativa en la pasividad del hijo, de modo que este percibe de forma correcta la meta que persigue su madrey muestra menos pasividad en la interacción.

Por su parte, el nivel de representación de la acción materna solo predice negativamente la pasividad del hijo/a en el vídeo, al igual que en Rodríguez et al. (2011). Por tanto, una descripción compleja del comportamiento de la madre no influye en las acciones que esta desarrolla, pero sí influye en las del hijo/ a. Téngase en cuenta que un nivel alto de representación implica que la madre describe sus acciones en términos de las metas que quiere conseguir con las mismas. Esa forma de reflexionar sobre su conducta se transforma en indicios que llevan a que el niño comprenda mejor las intenciones de la madre y se muestre menos pasivo durante la interacción. En esta misma línea, las metas de normas, afecto y promoción del desarrollo que la madre dice perseguir, predicen de forma positiva la pasividad del menor, probablemente porque implican demasiado control sobre su comportamiento, lo que restringe las iniciativas del hijo/a durante la tarea. En suma, en las familias en riesgo psicosocial, se da un intercambio educativo entre madre-hijo en la realización de tareas conjuntas cuyos condicionantes negativos son el uso de metas y prácticas inadecuados, y los condicionantes positivos son el modo en que la madre se representa su acción en términos de metas por alcanzar y el grado en que el hijo/a "lee" adecuadamente los mensajes educativos de la madre. Estos últimos condicionantes capturan más la esencia del proceso de socialización y tienen capacidad predictiva sobre la calidad de la interacción, una vez examinado el efecto de las variables tradicionales (prácticas y metas). Por tanto, estudios futuros deben seguir investigando en esta línea.

Este estudio tiene varias implicaciones prácticas. En los programas de educación parental con familias en situacion de riesgo psicosocial es necesario tratar de disminuir las prácticas coercitivas y restrictivas, mejorar la verbalización que hace la madre de sus comportamientos para que alcance una clara orientación a metas, así como mejorar la "lectura" del hijo/a de los mensajes de la madre/padre. Todo ello ayudará a regular la interacción en las actividades cotidianas de la familia, con la finalidad de favorecer los ajustes interactivos y mejorar los procesos de aprendizaje, en la segunda infancia y pre-adolescencia. Por tanto, no solo es necesario cambiar los estilos educativos, sino que hay que prestar especial atención al complejo proceso de transmisión de los mensajes educativos por su influencia en la calidad de la interacción entre madre e hijo/a. 


\section{Agradecimientos}

Expresamos nuestro agradecimiento a los equipos de Servicios Sociales de los municipios de Tenerife y a todas las madres e hijos/as que participaron en este estudio. Este trabajo fue apoyado por el Ministerio de Economía y Competitividad (MINECO) y el Fondo Europeo de Desarrollo Regional (FEDER), mediante el proyecto PSI2015-69971-R.

\section{Referencias}

Álvarez, M., Rodrigo, M. J., \& Rodríguez, G. (2013). Predictibilidad de los mensajes educativos entre madres e hijos en familias en riesgo psicosocial. Infancia y Aprendizaje, 36(2), 219-229.

Azar, S. T. (2002). Child abuse. En M. Bornstein (Ed.), Handbook of parenting (vol. 4, pp. 361-388). Nueva York: Erlbaum.

Bernier, A., Calkins, S. D., \& Bell, M. A. (2016). Longitudinal associations between the quality of mother-infant interactions and brain development across infancy. Child Development, 87(4), 1159-1174. doi: 10.1111/cdev. 12518

Bousha, D. M., \& Twentyman, C. T. (1984). Mother-child interactional style in abuse, neglect, and control groups: Naturalistic observation in the home. Journal of Abnormal Psychology, 93, 106-114.

Barbot, B., Crossman, E., Hunter, S. R., Grigorenko, E. L., \& Luthar, S. S. (2014). Reciprocal influences between maternal parenting and child adjustment in a highrisk population: A 5-year cross-lagged analysis of bidirectional effects. American Journal of Orthopsychiatry, 84(5), 567-580.

Bugental, D. B., \& Grusec, J. (2006). Socialization processes. En W. Damon \& R. M. Lerner (Eds. de la serie) \& N. Eisenberg (Ed. del volumen), Handbook of child psychology: Vol. 3. Social, emotional and personality development (6a. ed., pp. 366-428). Hoboken, NJ: Wiley.
Cicchetti, D., \& Toth, S.L. (2015). A multilevel perspective on child maltreatment. En M. Lamb \& C. Garcia (Eds.), Handbook of child psychology and developmental science: Vol. 3. Socioemotional process (pp. 513-563). Hoboken, NJ: Wiley.

Conger, R. D., Wallace, L. E., Sun, Y., Simons, R. L., McLoyd, V. C., \& Brody, G. H. (2002). Economic pressure in African American families: A replication and extension of the family stress model. Developmental Psychology, 38(2), 179-93.

Crittenden, P. M. (1981). Abusing, neglecting, problematic, and adequate dyads: Differentiating by patterns of interaction. Merrill-Palmer Quarterly, 27, 1-18.

Egeland, B., Pianta, R., \& O'Brien, M. A. (1993). Maternal intrusiveness in infancy and child maladaptation in early school years. Development and Psychopathology, 5(3), 359-370.

Gauvain, M., \& Perez, S. M. (2007). The socialization of cognition. En J. Grusec \& P. Hastings (Eds.), Handbook of socialization: Theory and research (pp. 588-613). Nueva York: Guilford.

Grusec, J. E., \& Davidov, M. (2010). Integrating different perspectives on socialization theory and research: A domain-specific approach. Child Development, 81 (3), 687-709. doi: 10.1111/ j.1467-8624.2010.01426.x

Grusec, J. E., \& Goodnow, J. J. (1994). Impact of parental discipline methods on the child's internalization of values: A reconceptualization of current points of view. Developmental Psychology, 30, 4-19.

Grusec, J. E., \& Walters, G. C. (1991). Psychological abuse and childrearing belief systems. En R. H. Starr Jr. \& D. A. Wolfe (Eds.), The effects of child abuse and neglect: Issues and research (pp. 100-128). Nueva York: Guilford.

Hoffman, M. (1970). Moral development. En P. H. Mussen (Ed.), Carmichael's Manual of Child Psychology (Vol. 2, pp. 261-360). Nueva York: Wiley. 
Howes, P. W., Cicchetti, D., \& Toth, S. L. (2000). Affective, organizational, and relational characteristics of maltreating families: A system's perspective. Journal of Family Psychology, 14(1), 95-110.

Kuczynski, L. (2003). Beyond bidirectionality: Bilateral conceptual frameworks for understanding dynamicsin parent-child relations. En L. Kuczynski (Ed.), Handbook of dynamics in parent-child relations (pp. 3-24). Thousand Oaks, CA: Sage.

Kuczynski, L., \& De Mol, J. (2015). Dialectical models of socialization. En W. F. Overton \& P. C. M. Molenaar (Eds.), Handbook of child psychology and developmental science. Theory and method (Vol. 1, 7a. ed., pp. 323-368). Hoboken, NJ: Wiley.

Maccoby, E. E. (1992). The role of parents in the socialization of children: An historical overview. Developmental Psychology, 28, 1006-1017.

Maccoby, E. E. (2003). Dynamic viewpoints on parent-child relations: Their implications for socialization processes. Handbook of Dynamics in Parent-Child Relationships (pp. 439-452). Thousand Oaks, CA: Sage.

Martínez, I., \& García, J. F. (2007). Impact of parenting styles on adolescents' selfesteem and internalization of values in Spain. Spanish Journal of Psychology, 10(2), 338-348. doi: 10.1017/S113874160000660

Oldershaw, L., Walters, G., \& Hall, D. K. (1986). Control strategies and noncompliance in abusive mother-child dyads: An observational study. Child Development, 57, $722-732$.

Orozco-Hormaza, M., Sánchez-Ríos, H., \& Cerchiaro-Ceballos, E. (2012). Relación entre desarrollo cognitivo y contextos deinteracción familiar de niños que viven en sectores urbanos pobres. UniversitasPsychologica, 11(2), 427-440.

Trickett, P. K., \& Kuczynski, L. (1986). Children's misbehaviors and parental discipline strategies in abusive and nonabusive families. Developmental Psychology, $22,115-123$.
Radziszewska, B., \& Rogoff, B. (1988). Influence of adult and peer collaborators on children's planning skills. Developmental Psychology, 24, 840-848.

Rodrigo, M. J., Byrne, S., \& Rodríguez, B. (2013). Parenting styles and child well-being. En A. Ben-Arieh, I. Frønes, F. Casas \& J. E. Korbin (Eds.), Handbook of child well-being (pp. 1313-1319). Holland: Springer.

Rodrigo, M. J., Janssens, J. M. A. M., \& Ceballos, E. (1999). Do children's perceptions and attributions mediate the effects of mothers' child-rearing actions? Journal of Family Psychology, 13(4), 508-522.

Rodrigo, M. J., Janssens, J. M. A. M., \& Ceballos, E. (2001). Reasoning and action complexity: Sources and consequences on maternal childrearing behavior. International Journal of Behavioral Development, 25(1), 50-59. doi: 10.1080/01650250042000069

Rodrigo, M. J., Máiquez, M. L., Martín, J. C., \& Byrne, S. (2008). Preservación familiar. Un enfoque positivo para la intervención con familias. Madrid: Pirámide.

Rodriguez, G., Rodrigo, M. J., Janssens, J. M. A. M., \& Triana, B. (2011). Maternal conceptions of the parenting role and mother-child collaborative behaviors in at-risk context. European Journal of Developmental Psychology, 8(4), 389-402.

Rogoff, B. (1990). Apprenticeship of thinking. Nueva York: Oxford University Press.

Tabachnick, B. G., \& Fidell, L. S. (2007). Using multivariate statistics (5a. ed.). Boston, MA: Pearson Education.

Vallacher, R. R., \& Wegner, D. M. (1985). A theory of action identification. Hillsdale, NJ: Erlbaum.

\section{Notas}

* Artículo de investigación 